\title{
Optical Kerr-Effect in Fiber-Optic Brillouin Ring Laser Gyroscopes
}

\author{
S. Huang, L. Thévenaz, K. Toyama, B. Y. Kim, and H. J. Shaw
}

\begin{abstract}
The optical Kerr-effect in a reciprocal fiber-optic Brillouin ring laser gyroscope is investigated. It is found that the Kerr effect, as the major error source of the rotation rate measurement in this gyroscope, causes a bias of beat frequency with nonlinear dependence on rotation rate. A differential Stokes power of $1 \mathrm{~mW}$ inside the ring resonator is measured to produce a beat frequency bias of $69 \mathrm{~Hz}$, which agrees well with theoretical expectation.
\end{abstract}

\section{INTRODUCTION}

$\mathrm{T}$ HIS letter investigates the optical Kerr-effect in a Brillouin fiber-optic gyroscope (BFOG) which utilizes stimulated Brillouin scattering as the gain mechanism [1]-[3]. The advantage of the BFOG over other types of fiber-optic gyroscopes is its direct readout of rotation rate, measured as a beat frequency between counter-propagating lasing waves inside a fiber ring resonator. In BFOG systems previously reported, the frequency separation of two resonator modes was used as a constant beat frequency offset [1], [2] and the fluctuation of this offset due to, for example, temperature could be a major error source in the rotation rate measurement. On the other hand, the optical Kerr-effect (present in all BFOG's) is the main error source for the reciprocal BFOG system reported here, which uses the same resonator mode for both counter-propagating lasing waves [3]. The Kerr-effect has been investigated previously in interferometric fiber-optic gyroscopes (IFOG's) [4] and resonant fiberoptic gyroscopes (RFOG's) [5], [6]. This effect causes a non-reciprocity between the two counter-propagating lightwaves in the fiber coil, resulting in a spurious rotation signal. Various methods for reducing the Kerr-effect have been discussed also in the context of IFOG's [7], [8] and RFOG's [5], [9]. However, this letter represents the first detailed study of the Kerr-effect in a BFOG system. In the following, an all-fiber reciprocal BFOG is described first. Theoretical analysis of the beat frequency offset due to the Kerr-effect is presented next. Finally, measurements of the beat frequency of an experimental system are described and shown to agree with the theoretical analysis.

\section{RECIPROCAL BFOG SYSTEM}

Fig. 1 shows the schematic of the reciprocal BFOG system [3]. The system is made with nonpolarization-

Manuscript received November 3, 1992; revised December 24, 1992 This work was supported by Litton Systems, Inc.

The authors are with the Edward L. Ginzton Laboratory, Stanford University, Stanford, CA 94305.

IEEE Log Number 9207615. maintaining single-mode fibers, spliced together at points $S$. Pump light from a laser-diode-pumped $1.32 \mu \mathrm{m}$ Nd:YAG ring laser is split into waves $P_{1}^{\text {in }}$ and $P_{2}^{\text {in }}$ by the $3 \mathrm{~dB}$ coupler $C_{1} . P_{1}^{\text {in }}$ and $P_{2}^{\text {in }}$ are coupled to the fiber ring resonator through the coupler $C_{2}$, resulting in counterpropagating pump waves $P_{1}$ and $P_{2}$ in the ring. The states of polarization of pumps $P_{1}^{\text {in }}$ and $P_{2}^{\text {in }}$ at the coupler $C_{2}$ are adjusted by the polarization controllers PC1 and PC2 such that both $P_{1}$ and $P_{2}$ have the same eigen state of polarization in the ring.

The resonance of the resonator is locked to the pump frequency by a FM sideband technique, where the pump is modulated by a phase modulator PM2 and the throughput of the pump $P_{1}^{\text {in }}$ is tapped by the coupler $C_{3}$ and detected by the detector $D_{1}$. The detected signal is used by the stabilization circuits for adjusting the voltage applied to phase modulator PM1 in order to maintain the resonator at resonance for the pump $P_{1}$. This, in turn, ensures that the pump $P_{2}$ is also at resonance when the gyroscope is at rest, because $P_{1}$ and $P_{2}$ see the same resonant frequencies.

With resonance maintained, the power levels of $P_{1}$ and $P_{2}$ are enhanced greatly in the resonator and two Brillouin lasing waves $B_{1}$ and $B_{2}$ are excited in opposite directions to their respective pumps $P_{1}$ and $P_{2}$, when the power levels of $P_{1}$ and $P_{2}$ are above the Brillouin threshold. The Brillouin waves resonate, which selects their frequency and increases the efficiency of conversion of pump power to Brillouin power. The circulating Brillouin waves $B_{1}$ and $B_{2}$ are trapped by the coupler $C_{2}$ and combined by the coupler $C_{1}$ to produce a beat frequency output from detector $D_{2}$. When the gyroscope rotates, the resonant frequency seen by $P_{2}$ differs from that seen by $P_{1}$ due to the Sagnac effect. This "pump walk-off effect" or resonator detuning from resonance for $P_{2}$ reduces the power levels of $P_{2}$ and $B_{2}$ in the resonator. This limits the maximum measurable rotation rate because $B_{2}$ eventually vanishes once the power of $P_{2}$ becomes lower than the Brillouin threshold. At this point, the beat frequency is also lost.

\section{Beat Freouency Offset Induced by KERR-EFFECT}

When the BFOG system is operating properly, there are four circulating waves inside the ring: two pump waves $P_{1}$ and $P_{2}$, and two Brillouin waves $B_{1}$ and $B_{2}$, with their powers also represented by the same symbols $P_{1}, P_{2}, B_{1}$, and $B_{2}$, respectively. The nonlinear index seen by $B_{1}$ 


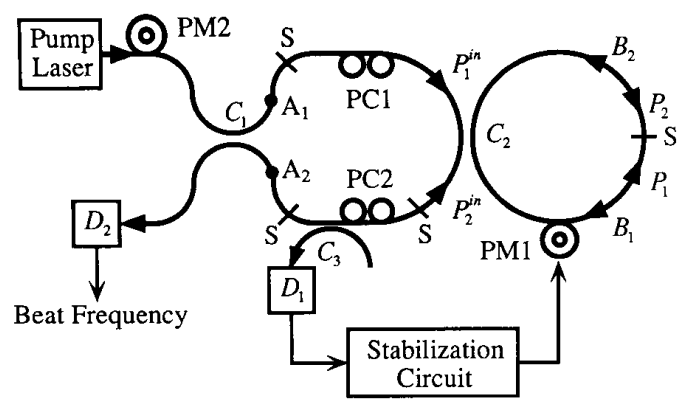

Fig. 1. Schematic diagram of a Brillouin fiber-optic gyroscope. $P_{1}$ and $P_{2}$ : pump waves; $B_{1}$ and $B_{2}$ : Brillouin waves; $D$ : detector, $C$ : coupler, PC: polarization controller, PM: phase modulator, and $S$ : splice.

through the Kerr-effect is given by

$$
\delta n_{B_{1}}=\alpha n_{2}\left(B_{1}+2 B_{2}+2 P_{1}+2 P_{2}\right) / A_{\text {eff }}
$$

where $n_{2}$ is the nonlinear index coefficient, $A_{\text {eff }}$ is the effective fiber-core area, and $\alpha$ accounts for polarization effects, ranging from 1 for linear polarization to $2 / 3$ for circular polarization [10]. Because the eigen state of polarization in a non-polarization-maintaining fiber resonator is elliptical in general, $\alpha$ should be between 1 and $2 / 3$ for the current system. There is a factor of two difference in the coefficients between the first term and the remaining three terms in (1) because the first term is the self phase modulation and the others are the cross phase modulation terms for $B_{1}$. Note that only the first two terms exist in the IFOG [7] and RFOG [5]. The nonlinear index seen by $B_{2}$ is similarly given by

$$
\delta n_{B_{2}}=\alpha n_{2}\left(2 B_{1}+B_{2}+2 P_{1}+2 P_{2}\right) / A_{\text {eff }}
$$

where the self phase modulation term is now the second one. The differential nonlinear index change $\Delta n_{B}=\delta n_{B_{2}}$ $-\delta n_{B_{1}}$ between $B_{1}$ and $B_{2}$ results in a beat frequency offset. Including both the Sagnac effect and the Kerreffect, the beat frequency between $B_{1}$ and $B_{2}$ can be written as

$$
\Delta f_{B}=f_{B 2}-f_{B 1}=S \Omega+\eta \Delta B .
$$

The first term in (3) represents the Sagnac effect with scale factor $S$ and rotation rate $\Omega$, the positive sign of which corresponds to the clockwise rotation in Fig. 1, and the second term represents the beat frequency offset due to the index difference caused by the Kerr-effect with power imbalance $\Delta B=B_{2}-B_{1}$ and coefficient $\eta=$ $\left(f \alpha n_{2}\right) /\left(n A_{\text {eff }}\right)$, where $f$ is the average frequency of the Brillouin waves and $n$ is the effective refractive index of the fiber. The second term $\eta \Delta B$ causes a spurious rotation rate measurement. The calculated value of $\eta$ for the current system ranges from $51 \mathrm{~Hz} / \mathrm{mW}$ for a circular polarization to $76 \mathrm{~Hz} / \mathrm{mW}$ for a linear polarization, where $n_{2}=3.2 \times 10^{-16} \mathrm{~cm}^{2} / \mathrm{W}[11]$ and $A_{\text {eff }}=\pi \times 4.6^{2} \mu \mathrm{m}^{2}$ are used. Note that in (3) the pump waves are canceled out and the beat frequency offset is only proportional to the power imbalance $\Delta B=B_{2}-B_{1}$.

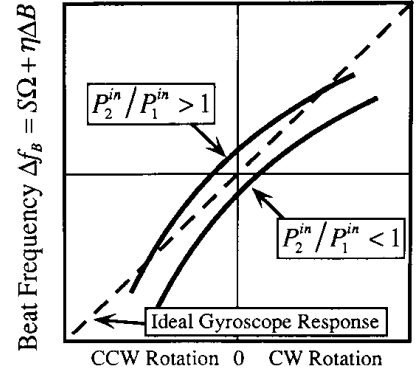

Rotation Rate $\Omega$

Fig. 2. Theoretically expected dependence of the beat frequency on rotation rate under Kerr-effect. $P_{2}^{\text {in }} / P_{1}^{\text {in }}$ is the ratio of the input pump powers.

The power imbalance $\Delta B$ could be caused by two factors. The first is initial power imbalance between the input pumps $P_{1}^{\text {in }}$ and $P_{2}^{\text {in }}$ at the coupler $C_{2}$, possibly due to an imperfect splitting ratio of the coupler $C_{1}$ and/or unequal losses in the fiber arms from the coupler $C_{1}$ to the coupler $C_{2}$. The sign of the power imbalance $\Delta B=$ $B_{2}-B_{1}$ is equal to the sign of $P_{2}^{\text {in }} / P_{1}^{\text {in }}-1$. The second is the pump walk-off effect, where the levels of $P_{2}$ and $B_{2}$ are reduced at high rotation rate, making $\Delta B$ negative at high rotation rate.

The effects of these two factors are schematically shown in Fig. 2. The ideal gyroscope response due only to the Sagnac effect is the dotted straight line passing through the origin. When the Kerr-effect is included, the gyroscope response is either shifted upward for $P_{2}^{\text {in }} / P_{1}^{\text {in }}>1$ or downward for $P_{2}^{\text {in }} / P_{1}^{\text {in }}<1$ and it is also bent downward at high rotation rate because of the pump walk-off effect. The response is also truncated in the figure, indicating the disappearance of the beat signal due to the pump walk-off effect at high rotation rate.

\section{EXPERIMENT}

The beat frequency of the BFOG system of Fig. 1 was measured as a function of rotation rate. The length, free spectrum range, finesse, and linewidth (FWHM) of the resonator are $27 \mathrm{~m}, 7.5 \mathrm{MHz}, 75$, and $100 \mathrm{kHz}$, respectively. The balance of the input pumps $P_{2}^{\text {in }} / P_{1}^{\text {in }}$ was adjusted by introducing bending losses at points $A_{1}$ and $A_{2}$ in Fig. 1. The input pump powers $P_{1}^{\text {in }}$ and $P_{2}^{\text {in }}$ were measured using a non-invasive clamp-on microbending coupler (courtesy of Raynet Corp.). In order to magnify the beat frequency offset, which is much smaller than the beat frequency itself, the beat frequency due to rotation is subtracted from the total measured beat frequency $\Delta f_{B}$ in plotting the experimental results. In Fig. 3 the vertical axis is $\Delta f_{B}-S \Omega$, where the scale factor $S=0.966$ $(\mathrm{kHz} / \mathrm{deg} / \mathrm{sec})$ is determined from the linear fit to the data at low rotation rate. Thus, Fig. 3 shows the difference between the curved response and the dotted straight line in Fig. 2.

Three sets of data are shown in Fig. 3, corresponding to three input pump ratios $P_{2}^{\text {in }} / P_{1}^{\text {in }}=1.89,1.43$, and 0.79 . 


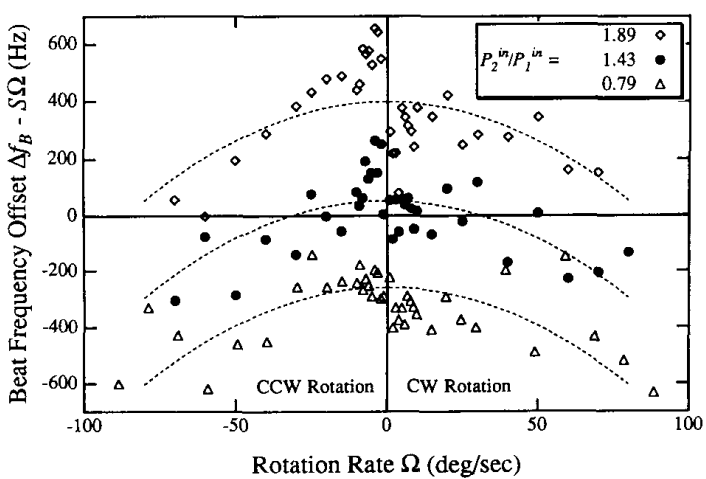

Fig. 3. Measured beat frequency bias as a function of rotation rate. Three data sets are shown at input pump power ratios $P_{2}^{\text {in }} / P_{1}^{\text {in }}=1.89$, 1.43 , and 0.79 .

The beat frequency is lost at about $90^{\circ} / \mathrm{s}$ because of the pump walk-off effect. Although the data points are scattered somewhat, presumably due to fluctuating power of the circulating Brillouin waves, which modulates the beat frequency offset through the Kerr-effect, the following observations can be made. First the data sets shift downward as the input pump ratio $P_{2}^{\text {in }} / P_{1}^{\text {in }}$ changes from 1.89 to 0.79 . Second, the data sets bend downward at high rotation rate. These two observations agree with the expectations based on the Kerr-effect, as schematically shown in Fig. 2.

Fig. 4 shows the beat frequency offset $\Delta f_{B}-S \Omega$ at $\Omega=0$ as a function of the power imbalance $\Delta B$. The power imbalance $\Delta B=B_{2}-B_{1}$ is determined by calculating the circulating Brillouin power $B_{j}$ in the ring from the measured input pump power $P_{j}$ using a formula based on Brillouin fiber ring laser theory [12]: $B_{j}=$ $P_{\text {th }}\left\{\sqrt{P_{j}^{\text {in }} / P_{\text {th }}^{\text {in }}}-1\right\},(j=1,2)$, where $P_{\text {th }}$ and $P_{\text {th }}^{\text {in }}$ are the circulating pump power threshold and the input pump power threshold which are 0.37 and $21 \mathrm{~mW}$, respectively, in the current system. The vertical error bars in Fig. 4 show the fluctuation of the experimental beat frequencies as previously mentioned. The slope of the best linear fit to the data points is $69 \mathrm{~Hz} / \mathrm{mW}$, which lies between the theoretical limits of 51 and $76 \mathrm{~Hz} / \mathrm{mW}$. This agreement further verifies that the beat frequency offset is caused by the Kerr-effect.

\section{CONCLUSIONS}

The large Kerr coefficient implies a requirement on the power balance between the two internal Brillouin waves to an accuracy of a few milliwatts for low grade to a few nanowatts for high grade gyroscopes. For balancing the two input pump powers, a feed-back control scheme developed for the RFOG [9] can be adopted. To avoid the

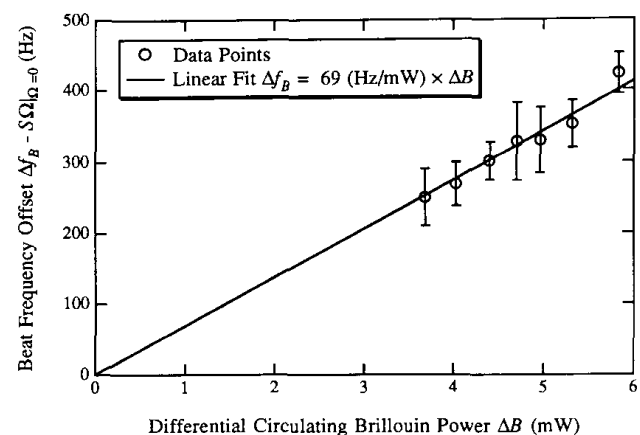

Fig. 4. Measured dependence of the beat frequency bias on the differential Brillouin power inside the resonator. The straight line is the best linear fit with slope of $69 \mathrm{~Hz} / \mathrm{mW}$.

Brillouin power unbalance caused by the pump walk-off effect, we propose a symmetrical resonance stabilization, which taps both input pumps $P_{1}^{\text {in }}$ and $P_{2}^{\text {in }}$ and places the pump frequency at midway between the two resonant frequencies seen by $P_{1}$ and $P_{2}$, so that the Brillouin powers $B_{1}$ and $B_{2}$ are balanced even at high rotation rates. This approach is presently being studied.

\section{REFERENCES}

[1] F. Zarinetchi, S. P. Smith, and S. Ezekiel, "Stimulated Brillouin fiber-optic laser gyroscope," Opt. Lett., vol. 16, pp. 229-231, 1991.

[2] R. K. Kadiwar and I. P. Giles, "Optical fiber Brillouin ring laser gyroscope," Electron. Lett., vol. 25, pp. 1729-1731, 1989.

[3] S. Huang, K. Toyama, P.-A. Nicati, L. Thévenaz, B. Y. Kim, and H. J. Shaw, "Brillouin fiber optic gyro with push-pull phase modulator and synthetic heterodyne detection," in Proc. SPIE, Fiber Optic and Laser Sensors $X$, vol. 1795, Boston, MA, Sept. 8-11, 1992.

[4] S. Ezekiel, J. L. Davis, and R. Hellwarth, "Intensity dependent nonreciprocal phase shift in a fiberoptic gyroscope," in Proc. First Internat. Conf. Fiber-Optic Rotation Sensors and Related Technologies, Springer-Verlag, New York, 1982, pp. 332-336.

[5] K. Iwatsuki, K. Hotate, and M. Higashiguchi, "Kerr effect in an optical passive ring-resonator gyro," J. Lightwave Technol., vol. LT-4, pp. 645-651, 1986.

[6] K. Takiguchi and K. Hotate, "Partially digital-feedback scheme and evaluation of optical Kerr-effect induced bias in optical passive ring-resonator gyro," IEEE Photon. Technol. Lett., vol. 3, pp. 679-681, 1991.

[7] R. A. Bergh, H. C. Lefevre, and H. J. Shaw, "Compensation of the optical Kerr effect in fiber-optic gyroscopes," Opt. Lett., vol. 7, pp. $282-284,1982$

[8] R. A. Bergh, B. Culshaw, C. C. Cutler, H. C. Lefèvre, and H. J. Shaw, "Source statistics and the Kerr effect in fiber-optic gyroscopes," Opt. Lett., vol. 7, pp. 563-565, 1982.

[9] K. Takiguchi and K. Hotate, "Method to reduce the optical Kerr-effect-induced bias in an optical passive ring-resonator gyro," IEEE Photon. Technol. Lett., vol. 4, pp. 203-206, 1992.

[10] R. A. Bergh, Ph.D. dissertation, Stanford University, Stanford, CA, 1983 , ch. 4.

[11] G. P. Agrawal, Nonlinear Fiber Optics. New York: Academic, 1989 , p. 183.

[12] P. Bayvel and I. P. Giles, "Evaluation of performance parameters of single-mode all-fiber Brillouin ring lasers," Opt. Lett., vol. 14, pp. $581-583,1989$. 